\title{
Short-term memory in Down syndrome: Applying the working memory model
}

\author{
Christopher Jarrold and Alan D. Baddeley \\ Centre for the Study of Memory and Learning, Department of Psychology, University of Bristol
}

\begin{abstract}
This paper is divided into three sections. The first reviews the evidence for a verbal short-term memory deficit in Down syndrome. Existing research suggests that short-term memory for verbal information tends to be impaired in Down syndrome, in contrast to short-term memory for visual and spatial material. In addition, problems of hearing or speech do not appear to be a major cause of difficulties on tests of verbal short-term memory. This suggests that Down syndrome is associated with a specific memory problem, which we link to a potential deficit in the functioning of the 'phonological loop' of Baddeley's (1986) model of working memory. The second section considers the implications of a phonological loop problem. Because a reasonable amount is known about the normal functioning of the phonological loop, and of its role in language acquisition in typical development, we can make firm predictions as to the likely nature of the short-term memory problem in Down syndrome, and its consequences for language learning. However, we note that the existing evidence from studies with individuals with Down syndrome does not fit well with these predictions. This leads to the third section of the paper, in which we consider key questions to be addressed in future research. We suggest that there are two questions to be answered, which follow directly from the contradictory results outlined in the previous section. These are 'What is the precise nature of the verbal short-term memory deficit in Down syndrome', and 'What are the consequences of this deficit for learning'. We discuss ways in which these questions might be addressed in future work.
\end{abstract}

Keywords - Down syndrome, verbal short-term memory, phonological loop, working memory

\section{Outline}

This paper has three aims. First, to review the evidence for a verbal short-term memory deficit in Down syndrome, and in doing so to consider whether any observed deficit really reflects an underlying memory difficulty, or is instead caused by other factors. Second, to explore the possible implications of a verbal short-term memory deficit for individuals with Down syndrome, and finally, to highlight key questions that remain to be addressed by future work in this area. This paper draws heavily on material covered in an earlier paper published in Down Syndrome Research and Practice (Jarrold, Baddeley \& Phillips, 1999), and the reader is referred to this previous account for a fuller treatment of many of the issues raised here.

\section{A Review}

Short-term memory is the ability to 'hold in mind' information. For example, one might try to remember the string of digits in a telephone number while moving to the phone and dialling. It differs from long-term memory, where information, although not necessarily forgotten, is not always consciously available until recalled. Verbal shortterm memory refers to the ability to maintain verbal items such as words or digits, and is most often assessed using 'span' paradigms in which individuals are asked to remember increasing numbers of words or digits. The maximum number of items that an individual can remember, in correct order, is their span. Adults typically have a span of around seven items (Miller, 1956), but among individuals with Down syndrome verbal short-term memory spans are usually considerably lower than this.

In fact many studies have shown that individuals with Down syndrome have lower verbal short-term memory spans than would be predicted by their level of verbal ability or their general level of intellectual function. For example, Kay-Raining Bird and Chapman (1994) compared the digit spans of a group of 47 individuals with Down syndrome and a corresponding number of typically developing children of an equivalent level of intellectual functioning. Among these controls digit spans ranged from 3 to 6, with a span of 5 being most common. Although 1 of the indi- 
viduals with Down syndrome achieved a digit span of 6 , the average level of performance was considerably lower than that seen among controls; the most common span for individuals with Down syndrome was 4 and a number of individuals had a span of 2 .

This study illustrates two important aspects of verbal shortterm memory in Down syndrome. The first is that, on average, verbal spans tend to be lower than one would expect given individuals' other abilities (see also, Bower \& Hayes, 1994; Jarrold \& Baddeley, 1997; Jarrold, Baddeley \& Hewes, 1999, 2000; MacKay \& McDonald, 1976; Marcell \& Cohen, 1992; Marcell, Harvey \& Cothran, 1988; Marcell, Ridgeway, Sewell \& Whelan, 1995; Marcell \& Weeks, 1988; McDade \& Adler, 1980). However, it is also important to note that not all individuals with Down syndrome have low verbal short-term memory spans. Vallar and Papagno (1993) report the case of a young woman with Down syndrome, who, in addition to being fluent in 3 languages, had a perfectly normal verbal short-term memory span.

It is also worth noting that the problems experienced by the majority of individuals with Down syndrome on tests of verbal short-term memory are relatively specific, in that they do not appear to extend to all aspects of short-term memory. Short-term memory can be assessed for visuospatial information, as well as for verbal material; typically using a 'Corsi span' test (after Corsi, cited in Milner, 1971). In this task individuals watch while the tester taps out a series of different spatial locations on a board, and then have to repeat the sequence in the correct order themselves. As with digit span, the number of items to be remembered can be gradually increased to determine the maximum level of an individual's memory ability. The majority of studies which have contrasted digit and Corsi span among individuals with Down syndrome, in some cases relative to appropriate controls, have shown that individuals with Down syndrome are impaired only on digit span (Azari et al., 1994; Jarrold \& Baddeley, 1997; Jarrold, Baddeley \& Hewes, 1999; Wang \& Bellugi, 1994; though see Haxby, 1989; Vicari, Carlesimo \& Caltagirone, 1995). In other words, the individuals' visuo-spatial short-term memory is relatively unimpaired, in contrast to their verbal short-term memory performance.

However, this, in itself, does not necessarily prove that individuals with Down syndrome have a specific verbal shortterm memory problem. Their relatively poor performance on digit and word span tasks might arise for reasons which have comparatively little to do with memory. One obvious explanation for poor verbal short-term memory performance is that hearing difficulties, which are known to be relatively common in Down syndrome (Davies, 1996), affect individuals' ability to properly encode the items being presented. Verbal short-term memory tasks typically involve auditory presentation of the to-be-remembered items, and clearly if one has difficulty in hearing and identifying these items then one's memory for them is likely to be severely reduced.
This issue has been addressed in a number of elegant studies carried out by Marcell and colleagues. Marcell and Armstrong (1992) found that visual presentation of digits in a span task did not improve performance among individuals with Down syndrome, which contrasts with what would be expected if hearing difficulties were affecting the encoding of stimuli. Marcell, Harvey and Cothran (1988) found that presenting auditory stimuli through headphones also had no beneficial effect on the short-term memory performance of individuals with Down syndrome. Although this manipulation was designed to assess individuals' susceptibility to 'distractibility' in verbal short-term memory tasks, one might expect headphones to produce a higher quality auditory signal than normal speech. Finally, Marcell and Cohen (1992) found that direct measures of individuals' hearing ability did not predict their performance on a digit span task (see also Jarrold \& Baddeley, 1997). Again this is at odds with what one would expect if hearing difficulties were a major constraint on verbal short-term memory in Down syndrome. Having said this, Marcell and Cohen (1992) did find that the extent of hearing loss was related to the time taken by individuals with Down syndrome to identify digits, which may have some indirect effect on verbal short-term memory performance (Rabbitt, 1968).

A second possible explanation of verbal short-term memory difficulties in Down syndrome concerns problems of spoken articulation, which again are known to be associated with the condition (Dodd, 1975; Gibson, 1978). In word and digit span tasks, individuals typically give their response by repeating the presented list in full. It seems intuitively likely that an individual with difficulty in planning and/or executing a sequenced spoken response would show reduced verbal short-term memory spans as a result of these problems. Indeed work by Cowan and his colleagues (e.g., Cowan, Day, Saults, Keller, Johnson \& Flores, 1992) has shown that the time taken by typically developing individuals to produce their response in a verbal short-term memory task has a direct effect on their memory performance.

Once again Marcell and colleagues have done much of the work aimed at addressing this particular issue. In two studies Marcell and Weeks (1988) adapted digit and word span tasks so as to remove the need for individuals with Down syndrome to give a full spoken response at recall. In their first study, individuals recreated the to-be-remembered list in a digit span task by ordering a series of numbered blocks. In their second study, individuals gave their response to a word span task by pointing to pictures of the presented words in the correct serial order. In neither case did these manipulations improve the performance of individuals with Down syndrome relative to the standard recall procedure. If removing the need for a spoken response does not remove the difficulty with the task, then clearly this suggests that problems of speech are not a major factor in determining verbal short-term memory performance. A similar conclusion follows from the results of a recent study from our own research group. Jarrold et al. (2000) found that individuals with Down syndrome had lower verbal short-term memory spans than a comparison group of individuals with moder- 
ate learning difficulties, despite being able to repeat pairs of words in an articulation test at a similar rate to these controls. Again this implies that poor verbal short-term memory is not primarily due to problems of spoken articulation in Down syndrome.

In sum, individuals with Down syndrome tend to perform relatively poorly on tests of verbal-short term memory; a problem which appears to be specific to verbal tasks and does not extend to all short-term memory tests. Although one might well expect that problems of hearing and speech, which are associated with Down syndrome, are likely to be the cause of much of this difficulty, there is actually relatively little evidence to support this suggestion. Of course, this raises the question of what exactly is causing the relatively poor verbal short-term memory performance seen among individuals with Down syndrome? One possible answer is that there really is a fundamental shortterm memory problem associated with Down syndrome. A number of authors have advanced this view (e.g., Broadley, MacDonald \& Buckley, 1995; Comblain, 1996; Das \& Mishra 1995; Hulme \& Mackenzie, 1992; Jarrold \& Baddeley, 1997; Kay-Raining Bird \& Chapman, 1994; Mackenzie \& Hulme, 1987; Varnhagen, Das \& Varnhagen, 1987; Wang \& Bellugi 1994), and have tended to do so with reference to Baddeley and Hitch's 'working memory model'.

The working memory model, outlined initially by Baddeley and Hitch (1974) and refined by Baddeley (1986), represents an attempt to explain and account for a range of phenomena associated with short-term memory performance in both typically and atypically functioning individuals. A key feature of the model is that it suggests that separate systems are dedicated to the short-term storage of verbal and visuo-spatial information. These are termed the 'phonological loop' and the 'visuo-spatial sketchpad' respectively. Broadly speaking there are three lines of evidence to support this account. First, the type of experimental manipulations which affect verbal short-term memory performance have little or no effect on visuo-spatial short-term memory performance, and vice versa (e.g., Brandimonte, Hitch \& Bishop, 1992). Second, studies that have examined the pattern of brain activity in individuals performing verbal and visuo-spatial short-term memory tests have suggested that different locations in the brain are associated with these two sets of tasks (see Baddeley, 1993). Finally, and in line with the previous point, there are cases of adult neuropsychological patients who have suffered brain damage which has had a selective effect on verbal rather than visuo-spatial short-term memory, and vice versa. All three lines of evidence point to the separateness of the short-term memory systems responsible for the storage of verbal as opposed to visuo-spatial information; a suggestion which itself is consistent with the possibility of a selective verbal short-term memory problem in Down syndrome. In other words, according to the working memory model, Down syndrome may be associated with some form of 'phonological loop' deficit.

\section{Implications}

The claim of a phonological loop deficit in Down syndrome amounts to more than a restatement of the data reviewed above, because it has, at least, two important implications. The first follows from our knowledge of the likely consequences of a phonological loop problem and the second from our understanding of the normal functioning of this system.

There is considerable evidence to suggest that the phonological loop plays an important role in the development of aspects of language, and in vocabulary acquisition in particular (see Baddeley, Gathercole \& Papagno, 1998 for a review). The first evidence for the role of the loop in vocabulary acquisition came from work with a neuropsychological patient - PV - known to have impaired phonological loop functioning. Baddeley, Papagno and Vallar (1988) studied PV's ability to associate known target words with two types of material: other known words or novel 'nonsense' word stimuli. They showed that while PV was perfectly able to learn associations between pairs of known words, her ability to pair words with nonsense words was drastically impaired. These data were seen as being consistent with the view that the phonological loop is involved in maintaining the novel phonological form of a newly experienced word during vocabulary learning. Subsequent work by Gathercole and her colleagues, among others, has shown that children's verbal short-term memory functioning relates to their ability to learn the phonological form of new word stimuli in vocabulary learning tasks (e.g., Gathercole, Hitch, Service \& Martin, 1997). These results imply that individuals with Down syndrome should have problems in learning the phonological form of words during vocabulary acquisition, given their apparent problems in verbal shortterm memory.

In addition to knowing something about the probable consequences of a phonological loop impairment, our knowledge of the workings of the loop has implications for the precise type of problem experienced by individuals with Down syndrome, which in turn has potential implications for intervention strategies. Evidence from work with typically functioning adults has led Baddeley (1986) to suggest that the loop can be divided into two components. One is a passive store, which maintains information in a phonological form, but which is subject to forgetting due to the decay of verbal memory traces. A second process - rehearsal, offsets this decay. By subvocally repeating to-be-remembered information an individual can prolong the maintenance of material within the phonological loop.

The importance of rehearsal is shown by the effects of blocking this process. If adults are asked to continually repeat a word out loud during a verbal short-term memory task, a process known as 'articulatory suppression' which blocks rehearsal, then spans are markedly impaired (Baddeley, Thomson \& Buchanan, 1975). In addition, an insight into the nature of rehearsal is shown by the fact that the faster an individual is able to rehearse, as indexed by the speed with which they can articulate words out loud, the 
greater their verbal short-term memory span (e.g., Baddeley, et al. 1975). This is thought to reflect the time-limited nature of the store within the phonological loop - the faster one rehearses the more words one can maintain in the store without any being subjected to forgetting. This is further reflected in the 'word length effect'; individuals show higher spans for words with a short spoken duration, which take relatively less time to rehearse, than for words with a longer spoken duration (Baddeley et al., 1975). Clearly, given the importance of rehearsal in determining phonological loop efficiency, it is possible that the problems experienced by individuals with Down syndrome in verbal short-term memory tasks are due to a deficit in this process. If this is the case, then one might be able to target interventions towards this problem, in the hope of improving rehearsal inefficiency. Indeed a number of studies have attempted to improve or train rehearsal in individuals with Down syndrome (Broadley \& MacDonald, 1993; Broadley, MacDonald \& Buckley, 1994; Comblain, 1994; Laws, MacDonald \& Buckley, 1996; Laws, MacDonald, Buckley \& Broadley, 1995; for more details on rehearsal training see Conners, Rosenquist and Taylor (2001).

The claim of a phonological loop deficit in Down syndrome therefore has implications for our understanding of Down syndrome, and for possible intervention strategies. However, it is important to note that the accounts outlined above, although theoretically plausible, are not fully supported by empirical evidence. First, it is far from clear that vocabulary abilities in Down syndrome are as poor as one would predict given the evidence of a phonological loop deficit and the known importance of the loop in vocabulary acquisition. For example, Rondal (1995) reports the case of Françoise, an individual with Down syndrome with poor verbal short-term memory, but relatively superior vocabulary and other language skills. More generally, although researchers are not fully agreed about exactly how strong vocabulary abilities are in Down syndrome, it appears that vocabulary tends to be relatively advanced in comparison to other aspects of language (see Chapman, 1995). This should not be the case if a phonological loop deficit were affecting vocabulary acquisition directly, leading Laws (1998) to suggest that "The link between vocabulary and phonological memory in Down syndrome is not as well established as it is in normal development" (p. 1121).

Second, although rehearsal is certainly an important component of typical phonological loop functioning, there is growing evidence to suggest that a problem in rehearsal may not be the prime cause of poor verbal short-term memory in Down syndrome. This is not to say that rehearsal is entirely unimpaired, simply that problems in rehearsal may not be the main reason why individuals with Down syndrome show lower verbal short-term memory spans than other individuals. In our recent work (Jarrold et al., 2000) we compared the verbal short-term memory spans of a group of individuals with Down syndrome and a comparison group of individuals with learning disability but without Down syndrome. In addition, individuals' speech rates were measured by asking them to repeat sequences made up of the words used in the memory task as rapidly as possible. This provides a measure, albeit an indirect one, of the speed with which individuals should be rehearsing these words in the memory task. The individuals with Down syndrome had lower memory spans than the comparison group, in line with the body of research outlined above, but both groups had comparable speech rates. This implies that the problems in memory seen in these individuals with Down syndrome are not due to slower, more inefficient rehearsal.

Of course this assumes that individuals with Down syndrome actually employ rehearsal to aid their verbal shortterm memory. In fact there are reasons for thinking that individuals with Down syndrome might be unlikely to use rehearsal at all. These follow from evidence that typically developing individuals do not begin to rehearse until around the age of 7 years (see Gathercole, 1998). Although somewhat controversial, according to this view one might not expect to see rehearsal emerge among individuals with Down syndrome until they reach the equivalent developmental level. To assess this possibility we carried out a further study, in which the size of the word length effect was assessed in a 'probed recall' task. As discussed above, poorer memory for words of a longer spoken duration - the word length effect - is potential evidence that rehearsal is occurring. However, a word length effect might also arise because a sequence of long words takes longer to say when it comes to providing the response to a short-term memory test (Cowan, et al., 1992). To counter this latter issue, memory was assessed in a task where three words were presented and individuals were prompted to remember either the first, the second, or the third word in the list. Under these conditions individuals with Down syndrome did not show a word length effect, suggesting that they were not rehearsing. Their overall level of memory was poorer than a comparison group, but importantly these control individuals also showed no word length effect in recall. This implies that a problem of rehearsal is not the main cause of the verbal short-term memory deficit in this instance, because both groups showed no evidence of rehearsal, and yet the individuals with Down syndrome still had lower verbal short-term memory spans.

\section{Key Questions for Future Work}

The previous section highlighted two implications of the claim that Down syndrome is associated with problems of phonological loop function, but also noted that both of these implications are not clearly supported by the existing evidence from studies of Down syndrome. A phonological loop deficit would predict impaired vocabulary acquisition, but it is not clear that vocabulary learning is particularly problematic for individuals with Down syndrome. Rehearsal is a key process in phonological loop functioning, but it does not appear that problems in rehearsal are the main reason why verbal short-term memory is poor in Down syndrome. The two questions outlined here for future research follow from these two unresolved issues. 
The first question is whether a phonological loop problem has consequences for learning in Down syndrome. Although the existing evidence suggests that vocabulary is a relatively strong aspect of language functioning, there are few, if any, direct studies of vocabulary learning in Down syndrome. Chapman, Kay-Raining Bird and Schwartz (1990) have examined 'fast mapping' abilities. This is the ability to learn or realise that an unfamiliar word must be paired with an unfamiliar object. Fast mapping is tested, for example, by showing individuals two familiar and one novel object, and asking them to point to the 'koob' (or some other unfamiliar term). Chapman et al. (1990) found that individuals with Down syndrome had no difficulty with this task. However, this kind of task does not assess the precise aspects of vocabulary acquisition that are thought to be supported by the phonological loop, as it does not really test individuals' ability to learn and remember the phonological form of the novel word. Studies of this latter form of word learning have been conducted with typically developing children (e.g., Gathercole et al., 1990; Gathercole, et al., 1997), and could be adapted for use with individuals with Down syndrome.

A second key question concerns the precise nature of the apparent phonological loop problem in Down syndrome. If it does not reflect a rehearsal deficit, or problems of hearing and speech, then what is its cause? As outlined above, the phonological loop consists of two processes or components - rehearsal and the phonological store. If the deficit is not primarily one of rehearsal, then one is left to conclude that it must reflect some form of impairment with the phonological store. There are two problems with this suggestion. The first is that it is a rather 'post-hoc' account, and the second is that, as yet, we have no clear and accepted method of measuring phonological store functioning or capacity. One possible way in which this might be done is to look at the rate at which individuals forget verbal information over time. If individuals with Down syndrome do not rehearse, then the rate at which they forget information should provide a reasonably direct index of the efficiency of their phonological store.

\section{Conclusions}

This paper began with a review of verbal short-term memory in Down syndrome. Taken together, the evidence suggests that individuals with Down syndrome do have relatively poor verbal short-term memory abilities (although there are certainly individual exceptions to this rule), and that these appear not to be caused by problems of hearing or speech. This suggests that there really is a fundamental short-term memory problem associated with the condition, which in terms of Baddeley and Hitch's working memory model would be viewed as a phonological loop deficit. Two implications of this claim were outlined. First, that this should predict problems of vocabulary acquisition in Down syndrome, and second, that the problem might well be one of inefficient or absent rehearsal. However, it appears that neither of these suggestions is fully supported by the exist- ing evidence, and future work is clearly needed to determine the precise causes and consequences of a phonological loop deficit in Down syndrome.

\section{Acknowledgements}

The authors are supported by a grant from the Medical Research Council to Alan Baddeley and Susan Gathercole. We are grateful to the Down Syndrome Educational Trust for their invite to present this paper at the 3rd International Conference on Language and Cognition in Down syndrome.

\section{Correspondence}

Chris Jarrold • Centre for the study of Memory and Learning, Department of Psychology, University of Bristol, 8 Woodland Road, Bristol, BS8 ITN, UK・E-mail: C.Jarrold@bristol.ac.uk

\section{References}

Azari, N. P., Horwitz, B., Pettigrew, K. D., Grady, C. L., Haxby, J. V., Giacometti, K. R. \& Schapiro, M. B. (1994). Abnormal pattern of cerebral glucose metabolic rates involving language areas in young adults with Down syndrome. Brain and Language, 46, 1-20.

Baddeley, A. D. (1986). Working Memory. Oxford: OUP.

Baddeley, A. D. (1993). Verbal and visual subsystems of memory. Current Biology, 3, 563-565.

Baddeley, A., Gathercole, S. \& Papagno, C. (1998). The phonological loop as a language learning device. Psychological Review, 105, 158-173.

Baddeley, A. D. \& Hitch, G. J. (1974). Working memory. In G. Bower (Ed.), The Psychology of Learning and Motivation (pp. 47-89). New York: Academic Press.

Baddeley, A., Papagno, C. \& Vallar, G. (1988). When longterm learning depends on short-term storage. Journal of Memory and Language, 27, 586-596.

Baddeley, A. D., Thomson, N. \& Buchanan, M. (1975). Word length and the structure of short-term memory. Journal of Verbal Learning and Verbal Behavior, 14, 575-589.

Bower, A. \& Hayes, A. (1994). Short-term memory deficits and Down's syndrome: A comparative study. Down's Syndrome: Research and Practice, 2, 47-50.

Brandimonte, M. A., Hitch, G. J. \& Bishop, D. V. M. (1992). Influence of short-term memory codes on visual image processing: Evidence from image transformation tasks. Journal of Experimental Psychology: Learning, Memory and Cognition, 18, 157-165.

Broadley, I. \& MacDonald, J. (1993). Teaching short term memory skills to children with Down's syndrome. Down's Syndrome: Research and Practice, 1, 56-62.

Broadley, I., MacDonald, J. \& Buckley, S. (1994). Are children with Down's syndrome able to maintain skills learned from a short-term memory training programme. Down's Syndrome: Research and Practice, 2, 116-122.

Broadley, I., MacDonald, J. \& Buckley, S. (1995). Working memory in children with Down's syndrome. Down's Syndrome: Research and Practice, 3, 3-8.

Chapman, R. S. (1995). Language development in children and adolescents with Down syndrome. In P. Fletcher \& 
B. MacWhinney (Eds.), Handbook of Child Language, Oxford: Blackwell.

Chapman, R. S., Kay-Raining Bird, E. \& Schwartz, S. E. (1990). Fast mapping of words in event contexts by children with Down syndrome. Journal of Speech and Hearing Disorders, 55, 761-770.

Comblain, A. (1994). Working memory in Down's syndrome: Training the rehearsal strategy. Down's Syndrome: Research and Practice, 2, 123-126.

Comblain, A. (1996). Auditivo-vocal short-term memory's functioning in Down's syndrome: Implication for the model of working memory. Approche Neuropsychologique Des Apprentissages Chez L'Enfant, 8, 137-147.

Conners, F.A, Rosenquist, C.J. \& Taylor, L.A. (2001) Memory training for children with Down syndrome. Down Syndrome Research and Practice, 7(1), 25-33.

Cowan, N., Day, L., Saults, J. S., Keller, T. A., Johnson, T. \& Flores, L. (1992). The role of verbal output time in the effects of word length on immediate memory. Journal of Memory and Language, 31, 1-17.

Das, J. P. \& Mishra, R. K. (1995). Assessment of cognitive decline associated with aging: A comparison of individuals with Down syndrome and other etiologies. Research in Development Disabilities, 16, 11-25.

Davies, B. (1996). Auditory disorders. In B. Stratford \& P. Gunn (Eds.), New Approaches to Down Syndrome (pp. 100-121). London: Cassell.

Dodd, B. (1975). Recognition and reproduction of words by Down's syndrome and non-Down's syndrome retarded children. American Journal of Mental Deficiency, 80, 306-311.

Gathercole, S. E. (1998). The development of memory. Journal of Child Psychology and Psychiatry, 39, 3-27.

Gathercole, S. E. \& Baddeley, A. D. (1990). The role of phonological memory in vocabulary acquisition: A study of young children learning new words. British Journal of Psychology, 81, 439-454.

Gathercole, S. E., Hitch, G. J., Service, E. \& Martin, A. J. (1997). Phonological short-term memory and new word learning in children. Developmental Psychology, 6, 966-979.

Gibson, D. (1978). Down's Syndrome: The Psychology of Mongolism. Cambridge: Cambridge University Press.

Haxby, J. V. (1989). Neuropsychological evaluation of adults with Down's syndrome: patterns of selective impairment in non-demented old adults. Journal of Mental Deficiency Research, 33, 193-210.

Hulme, C. \& Mackenzie, S. (1992). Working Memory and Severe Learning Difficulties. Hove: Lawrence Erlbaum Associates.

Jarrold, C. \& Baddeley, A. D. (1997). Short-term memory for verbal and visuo-spatial information in Down's syndrome. Cognitive Neuropsychiatry, 2, 101-122.

Jarrold, C., Baddeley, A. D. \& Hewes, A. K. (1999). Genetically dissociated components of working memory: Evidence from Down's and Williams syndrome. Neuropsychologia, 37, 637-651.

Jarrold, C., Baddeley, A. D. \& Hewes, A. K. (2000). Verbal short-term memory deficits in Down syndrome: A consequence of problems in rehearsal? Journal of Child Psychology and Psychiatry, 41, 233-244.
Jarrold, C., Baddeley, A. D. \& Phillips, C. (1999). Down syndrome and the phonological loop: The evidence for, and importance of, a specific verbal short-term memory deficit. Down Syndrome Research and Practice, 6, 61-75.

Kay-Raining Bird, E. \& Chapman, R. S. (1994). Sequential recall in individuals with Down syndrome. Journal of Speech and Hearing Research, 37, 1369-1380.

Laws, G. (1998). The use of nonword repetition as a test of phonological memory in children with Down syndrome. Journal of Child Psychology and Psychiatry, 39, 1119-1130.

Laws, G., MacDonald, J. \& Buckley, S. (1996). The effects of a short training in the use of a rehearsal strategy on memory for words and pictures in children with Down syndrome. Down's syndrome: Research and Practice, 4, 70-78.

Laws, G., MacDonald, J., Buckley, S. \& Broadley, I. (1995). Long-term maintenance of memory skills taught to children with Down's syndrome. Down's Syndrome: Research and Practice, 3, 103-109.

MacKay, D. N. \& McDonald, G. (1976). The effects of varying digit message structures on their recall by mongols and non-mongol subnormals. Journal of Mental Deficiency Research, 20, 191-196.

Mackenzie, S. \& Hulme, C. (1987). Memory span development in Down's syndrome, severely subnormal and normal subjects. Cognitive Neuropsychology, 4, 303-319.

Marcell, M. M. \& Armstrong, V. (1982). Auditory and visual sequential memory of Down syndrome and nonretarded children. American Journal of Mental Deficiency, $87,86-95$.

Marcell, M. M. \& Cohen, S. (1992). Hearing abilities of Down syndrome and other mentally handicapped adolescents. Research in Developmental Disabilities, 15, 533-551.

Marcell, M. M., Harvey, C. F. \& Cothran, L. P. (1988). An attempt to improve auditory short-term memory in Down's syndrome individuals through reducing distractors. Research in Developmental Disabilities, 9, 405-417.

Marcell, M. M., Ridgeway, M. M., Sewell, D. H. \& Whelan, M. L. (1995). Sentence imitation by adolescents and young adults with Down's syndrome and other intellectual disabilities. Journal of Intellectual Disability Research, 39, 215-232.

Marcell, M. M. \& Weeks, S. L. (1988). Short-term memory difficulties and Down's syndrome. Journal of Mental Deficiency Research, 32, 153-162.

McDade, H. L. \& Adler, S. (1980). Down syndrome and short-term memory impairment: A storage or retrieval deficit? American Journal of Mental Deficiency, 84, 561-567.

Miller, G. A. (1956). The magical number seven, plus or minus two: Some limits on our capacity for processing information. Psychological Review, 63, 81-97.

Milner, B. (1971). Interhemispheric differences in the localisation of psychological processes in man. Cortex, 27, 272-277.

Rabbitt, P. M. A. (1968). Channel capacity, intelligibility and immediate memory. Quarterly Journal of Experimental Psychology, 20, 241-248. 
Rondal, J. A. (1995). Exceptional Language Development in Down Syndrome. Cambridge: Cambridge University Press.

Vallar, G. \& Papagno, C. (1993). Preserved vocabulary acquisition in Down's syndrome: The role of phonological short-term memory. Cortex, 29, 467-483.

Varnhagen, C. K., Das, J. P. \& Varnhagen, S. (1987). Auditory and visual memory span: Cognitive processing by TMR individuals with Down syndrome or other etiologies. American Journal of Mental Deficiency, 91, 398-405.
Vicari, S., Carlesimo, A. \& Caltagirone, C. (1995). Shortterm memory in persons with intellectual disabilities and Down's syndrome. Journal of Intellectual Disability Research, 39, 532-537.

Wang, P. P. \& Bellugi, U. (1994). Evidence from two genetic syndromes for a dissociation between verbal and visual-spatial short-term memory. Journal of Clinical and Experimental Neuropsychology, 16, 317-322. 